\title{
Plasma optical shutter in ultraintense laser-foil interaction
}

\author{
W. Q. Wei, ${ }^{1,2}$ X. H. Yuan, ${ }^{1,2, a)}$ Y. Fang, ${ }^{1,2}$ Z. Y. Ge, ${ }^{3}$ X. L. Ge, ${ }^{1,2,4}$ S. Yang, ${ }^{1,2}$ Y. F. Li, ${ }^{5}$ G. Q. Liao, ${ }^{5}$ Z. \\ Zhang, ${ }^{5,2}$ F. Liu, ${ }^{1,2}$ M. Chen, ${ }^{1,2}$ L. Zhao, ${ }^{4}$ H. B. Zhuo, ${ }^{3,2}$ Y. T. Li, ${ }^{5,6,2}$ Z. M. Sheng, ${ }^{1,2,7}$ and J. Zhang ${ }^{1,2, b)}$ \\ ${ }^{1)}$ Key Laboratory for Laser Plasmas (Ministry of Education) and School of Physics and Astronomy, \\ Shanghai Jiao Tong University, Shanghai 200240, China \\ ${ }^{2)}$ Collaborative Innovation Center of IFSA (CICIFSA), Shanghai Jiao Tong University, Shanghai 200240, \\ China \\ ${ }^{3)}$ College of Science, National University of Defense Technology, Changsha 410073, \\ China \\ ${ }^{4)}$ State Key Laboratory of Surface Physics and Department of Physics, Fudan University, Shanghai 200433, \\ China \\ ${ }^{5)}$ Beijing National Laboratory of Condensed Matter Physics, Institute of Physics, Chinese Academy of Sciences, \\ Beijing 100190, China \\ 6) School of Physical Sciences, University of Chinese Academy of Sciences, Beijing 100049, \\ China \\ 7) SUPA, Department of Physics, University of Strathclyde, Glasgow G4 ONG, \\ United Kingdom
}

We report on a plasma optical shutter to reduce the intensity level of nanosecond-duration pedestal of the amplified spontaneous emission (ASE) using an ultrathin foil. The foil is ionized by the ASE prepulse and forms an expanding underdense preplasma, which enables the main laser pulse transmission, leading to an enhancement in temporal contrast. When such a plasma shutter is applied in front of an interested main target, the preplasma profiles are similar to that produced from single-layer reference target irradiated by highcontrast laser and can be finely tuned by varying the shutter thickness. Proton beam with significantly reduced divergence and higher flux density was measured experimentally using the double-foil design. The reduction in beam divergence is a characteristic signature of higher contrast laser production as a combined consequence of less target deformation and flatter sheath-acceleration field, as supported by the two-dimensional (2D) hydrodynamic and particle-in-cell simulations. The plasma shutter holds the promise to enhance the laser contrast and manipulate the preplasma conditions for applications in high-field-physics experiments.

PACS numbers: 52.38.Fz, 52.38.Ph

\section{INTRODUCTION}

With the rapid increase in achievable intensity of highpower laser systems, the effects of laser temporal contrast on laser-plasma interactions have received extensive attentions in recent years. ${ }^{1}$ For studies of advanced schemes of laser-driven ion acceleration such as the radiation pressure acceleration (RPA $)^{2,3}$ and laser breakout afterburner (BOA), ${ }^{4,5}$ and high-order harmonic generation (HHG) from solid surfaces, ${ }^{6,7}$ ultrahigh temporal contrast is an outstanding prerequisite in minimizing the deleterious effects of the target pre-expansion and deformation. The laser contrast also strongly influences properties of proton beam generated via the target-normalsheath-acceleration (TNSA) mechanism. ${ }^{8,9}$ It has been reported that improving the laser contrast will reduce the proton beam divergence. ${ }^{10,11}$ The smaller beam divergence could be a characteristic signature of higher contrast of the laser driver. ${ }^{12,13}$ The following two factors are responsible for less divergent proton beam production. Firstly, higher contrast laser will produce preplasma with smaller scalelengths both at the target front and

\footnotetext{
a) Electronic mail: xiaohui.yuan@sjtu.edu.cn.

b) Electronic mail: jzhang1@sjtu.edu.cn.
}

rear sides, which alters the generation and injection of energetic electrons into the target, resulting in the variation of sheath acceleration field distribution. ${ }^{14,15}$ Secondly, shock-induced target deformation is smaller or absent when launched by higher contrast laser. ${ }^{16,17}$ The spatialintensity distribution of proton beam can therefore be used to infer the conditions of laser pulse contrast when other laser and target parameters are the same.

To improve the laser contrast, several techniques have been employed, including the plasma mirror (PM), ${ }^{18}$ cross-polarized wave generation (XPW) ${ }^{19} \mathrm{OPCPA}^{20}$ and double $\mathrm{CPA}^{21}$ etc. Recent studies have shown that nanometer-thick ultrathin foil could serve as a transmissive plasma shutter to temporally shape the laser pulse based on the mechanism of relativistically induced transparency. ${ }^{22-25}$ The leading part of the laser pulse ionizes the foil to an expanding plasma such that the peak electron density $n_{e}$ is reduced and satisfies the critique of $n_{c}<n_{e}<\gamma n_{c}$. Here, $n_{c}\left(\mathrm{~cm}^{-3}\right)=1.11 \times 10^{21} / \lambda_{\mu m}^{2}$ is the critical density, $\gamma=\left(1+a_{0}^{2} / 2\right)^{1 / 2}$ is the relativistic factor, $a_{0}=8.5 \times 10^{-10} \lambda_{\mu m}\left[I\left(\mathrm{~W} / \mathrm{cm}^{2}\right)\right]^{1 / 2}$ is the normalized vector potential, $I$ is the laser intensity and $\lambda_{\mu m}$ is the wavelength in unit of micron. This plasma is relativistically transparent to the high-intensity peak while opaque to the low-intensity wings, resulting in a steeper-front pulse. The effectiveness in pulse shaping was investigated by indirectly measuring the variations of the proton beam 
properties, i.e. energy spectrum and directionality ${ }^{23}$ or directly measuring the phase and intensity envelope of the transmitted laser light. ${ }^{24}$ It should be noted that initial high contrast laser pulses have to be employed, which are critical to avoid the pre-expansion of nano-foil target into transparent, underdense plasma before the arrival of the main peak.

In this paper, we report a new regime of plasma shutter when the ultrathin foil expands into an underdense plasma $\left(n_{e}<n_{c}\right)$ subjected to the ASE pedestal for an initial low-contrast laser. We apply the shutter to a vacuum-gapped double-foil target (VGDF) design, i.e. the ultrathin shutter foil (SF) is placed before a main source foil (MF) sandwiched a vacuum gap, and measure the spatial-intensity distributions of multi-MeV TNSAprotons. The proton beam divergence is significantly reduced, implying its validity as a fast gating device. The increasing maximum proton energy with SF thickness further suggests the fine tunable preplasma is beneficial for enhancing proton acceleration.

\section{PLASMA SHUTTER MODEL AND HYDRODYNAMIC SIMULATIONS}

To evaluate the plasma generation and its evolution, one-dimensional (1D) hydrodynamic simulations were firstly performed using the code MULTI-fs. ${ }^{26}$ The MF is a $6.5 \mu \mathrm{m}$-thick aluminum foil and the SF is a carbon foil with the thickness ranging from $10 \mathrm{~nm}$ to $100 \mathrm{~nm}$. The vacuum gap is set to $30 \mu \mathrm{m}$. A single-layer target of MF is also simulated as reference. Step-profile ASE pedestal with intensity of $2 \times 10^{11} \mathrm{~W} / \mathrm{cm}^{2}$ on $\mathrm{SF}$ target is used, which corresponds to our initial laser condition, denoted as low contrast (LC).

Temporal evolutions of the electron density within $3 \mathrm{~ns}$ for a VGDF target of $100 \mathrm{~nm}$-thick SF and a reference target are shown in Fig. 1 (a) and (b). For a VGDF target (Fig. 1(a)), the laser ionizes the SF first and pushes it into the vacuum gap by the ablation pressure. The generated plasma expands in both directions. Whereafter, the MF front surface is also ionized to plasma state and expands towards the gap. Then a low-density preplasma with tens-of-micron thickness is formed before the MF. In comparison, for a reference target (Fig. 1(b)), the front surface is irradiated directly and ionized to plasma expanding into vacuum from the beginning.

To obtain the transverse distribution of electron density and its temporal evolution, 2D hydrodynamic simulations are further preformed using the code MULTI2D, which is developed with hydrodynamics in a twodimensional axial-symmetric configuration (cylindrical R-Z geometry). ${ }^{27}$ The laser has a Gaussian transverse profile with a $10 \mu \mathrm{m}$-diameter focal spot. To reduce the numerical noise and ensure convergence, the carbon thicknesses are increased to $1 \mu \mathrm{m}$ while the densities are reduced to maintain the areal density. Other target and laser parameters are the same as in the 1D simulations.
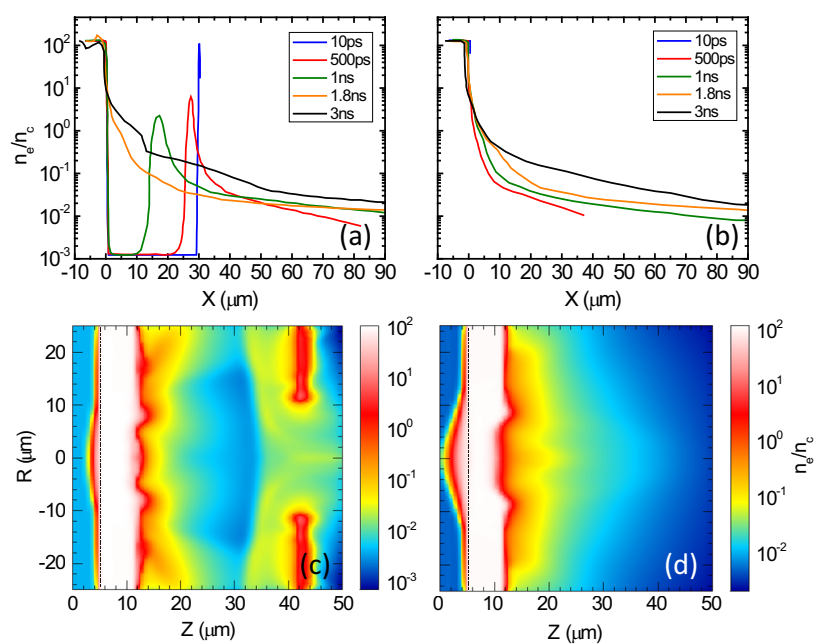

FIG. 1. (color online) Hydrodynamic simulations of: 1D electron spatial density profiles after ASE pulse irradiation at different times for (a) VGDF target of $100 \mathrm{~nm}$-thick SF and (b) single-layer reference target (with MF only). The front surface of MF is initially positioned at $x=0 \mu \mathrm{m}$. 2D target density distributions in cylindrical R-Z geometry at $t=1.8 \mathrm{~ns}$ for the corresponding targets of (c) VGDF target and (d) the reference target. The rear surface of MF is initially positioned at $z=5 \mu \mathrm{m}$ (shown in dash line). The ASE pulse is incident from the right-hand side.

Figures 1(c) and 1(d) are 2D electron density distributions at $t=1.8 \mathrm{~ns}$ for a VGDF target of $100 \mathrm{~nm}$-thick SF and a reference target, respectively. We see that the SF is broken-through and an aperture is formed. The laserlaunched shock wave propagates into the MF and deforms the rear surface by $1.2 \mu \mathrm{m}$ in depth. As similar as $1 \mathrm{D}$ results, the vacuum gap between the two foils is filled with low-density plasma of $0.01 n_{c}$ to $0.1 n_{c}$. In comparison, the shock breaks out the rear surface and causes obvious target deformation by $4.4 \mu \mathrm{m}$. The maximum deviations of the local normal directions of the curved rear surface with respect to the flat one give rise to an open angle of $26^{\circ}$ in comparison to $12^{\circ}$ for the VGDF target.

The thickness of SF, $L_{S F}$, was varied to investigate its influence on MF target deformation and preplasma formation as shown in Fig. 2. Different plasma distributions are produced and the densities in the vacuum gap are lower than that from the reference target. We see an approximately $15 \mu \mathrm{m}$-thick density plateau is developed in the front side of MF for thick SF $\left(L_{S F} \geq 50 \mathrm{~nm}\right)$. The plasma scalelength $\left(L=n_{e} /\left(d n_{e} / d z\right)\right)$ at the critical density along the main axis is $0.46 \mu \mathrm{m}$ for the VGDF target with $L_{S F}=100 \mathrm{~nm}$, in comparison with $1.1 \mu \mathrm{m}$ for the reference target. The density gradients at the rear side of MF are also increased. This suggests that the target deformation reduces with SF thickness.

We also simulated the interaction of the ASE pulse at 


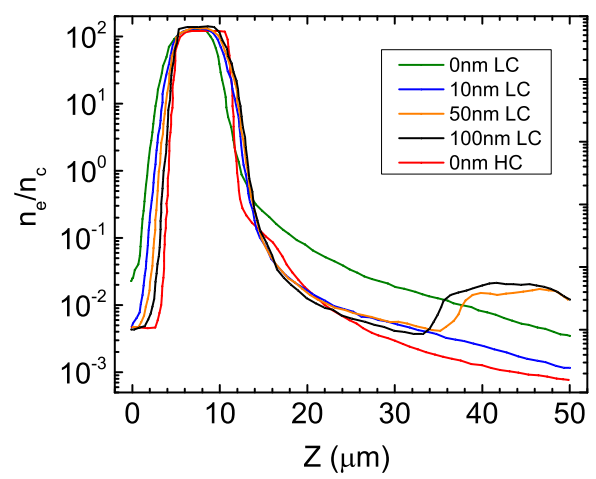

FIG. 2. (color online) Electron density profiles at $t=1.8 \mathrm{~ns}$ along the laser axis with different SF thickness in low (LC) or high contrast $(\mathrm{HC})$ cases. $L_{S F}=0 \mathrm{~nm}$ represents the reference target.

intensity of $1.5 \times 10^{9} \mathrm{~W} / \mathrm{cm}^{2}$, corresponding to a high contrast (HC) laser, with the reference target (red line in Fig. 2). The preplasma density profile is found to be similar to that of the VGDF target with $L_{S F}=10 \mathrm{~nm}$ under the LC condition. Simulations show that thicker SF or higher initial laser contrast will result in smaller plasma scalelength and efficiently reduce the influence of prepulses on MF. It turns out that a contrast-improved laser pulse is expected to be generated with controlled preplasma density distribution.

\section{EXPERIMENT}

To check the validity and effectiveness of the proposed plasma shutter in temporal contrast improvement, proton acceleration experiments were carried out using the 200 TW Ti:sapphire laser system in Shanghai Jiao Tong University. The schematic of experimental setup is shown in Fig. 3(a). The $800 \mathrm{~nm}, 25$ fs duration, p-polarized laser pulse was focused by an $f / 4$ off-axis parabolic mirror on $\mathrm{MF}$ at an incident angle of $9^{\circ}$. The energy on target was $1.5 \mathrm{~J}$, with a focal spot of $5 \mu \mathrm{m}$ full width at half maximum (FWHM) and 35\% energy contained within it. The calculated peak intensity was $1 \times 10^{20}$ $\mathrm{W} / \mathrm{cm}^{2}$ on $\mathrm{MF}$ and the approximate intensity on $\mathrm{SF}$ surface was $2 \times 10^{19} \mathrm{~W} / \mathrm{cm}^{2}$. The temporal contrast of 1.8 ns-long ASE pedestal to the main peak was measured to be $\sim 10^{-8}$, giving the ASE intensity on SF of $2 \times 10^{11}$ $\mathrm{W} / \mathrm{cm}^{2}$. When a plasma mirror system is inserted into the beam line, the contrast is improved by two orders of magnitude with an energy throughput of $70 \% .{ }^{11}$ The corresponding ASE intensity is reduced to $1.5 \times 10^{9}$ $\mathrm{W} / \mathrm{cm}^{2}$ on SF target.

Figure 3(b) shows sketch of the target assembly. A $30 \mu \mathrm{m}$-thick glass was employed as the spacer to separate and support both foils. The SF is commercial carbon foil widely used in the community of transmission

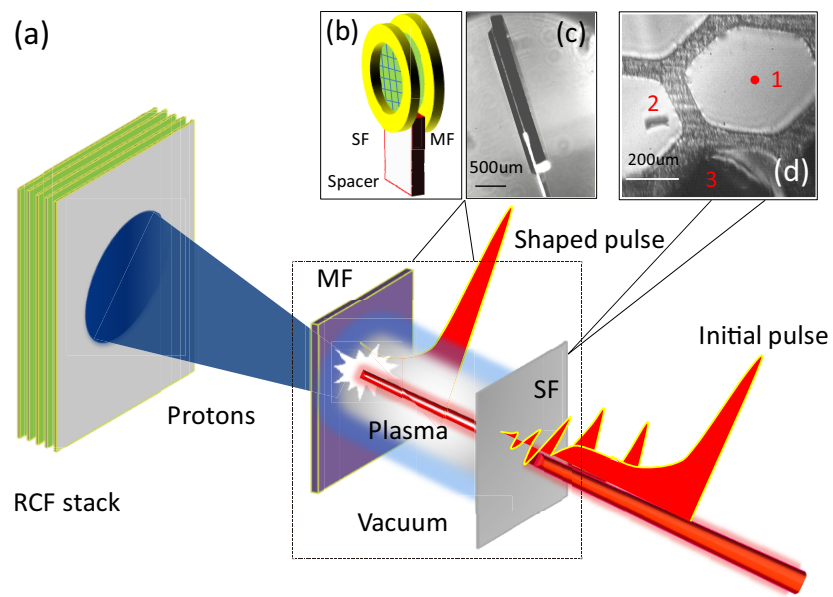

FIG. 3. (color online) (a) The schematic of experimental setup. The diagram in black dotted box denotes the optical plasma shutter design. (b) The sketch of target assembly. (c) The side-view image of the target. (d) The face-on image of the target. The cell 1 is the laser focus area, cell 2 is a contaminated area with dust and cell 3 is a broken area. The red dot indicates the laser focus position.

electron microscope. The foil has good quality (flatness and mechanical strength) due to the support by a $3 \mathrm{~mm}-$ diameter fine grid with $500 \mu \mathrm{m}$ period. The MF is floated on a $5 \mathrm{~mm}$-diameter washer with a $3 \mathrm{~mm}$ hole. The two frames were mounted face to face on the spacer in a U-shape, forming the $30 \mu \mathrm{m}$ vacuum gap. It allows the target assembly sustained during the vacuum chamber pumping. The side-view image of this target assembly monitored with a CW backlighter before shot is shown in Fig. 3(c). The gap and the two parallel frames are clearly seen. A high-magnification microscope was used at the target front side to aid the target alignment and monitor the SF surface appearance. Figure 3(d) shows a section of the target. We can see the cell 1 has relatively good quality. The laser was focussed through this cell to the MF. In comparison, cell 2 and cell 3 have been contaminated by the dust or broken.

Stacks of radiochromic film (RCF: Gafchromic ${ }^{\circledR}$ film, HD-V2) were used to record the proton beam spatialintensity distributions at coarse energy steps. The stack was positioned $40 \mathrm{~mm}$ from the target along the normal direction in the rear side, which was wrapped with $13 \mu \mathrm{m}$ $\mathrm{Al}$ foil to shield from direct laser radiation, target debris and low-energy ions.

\section{RESULTS AND DISCUSSION}

Figures 4(a) and 4(b) show the typical spatial-intensity distributions of $1.2 \mathrm{MeV}$ proton beams from the reference target and VGDF target $\left(L_{S F}=10 \mathrm{~nm}\right)$ under LC laser irradiation, respectively. The diameter of proton beam from the VGDF target is nearly half of that from the 

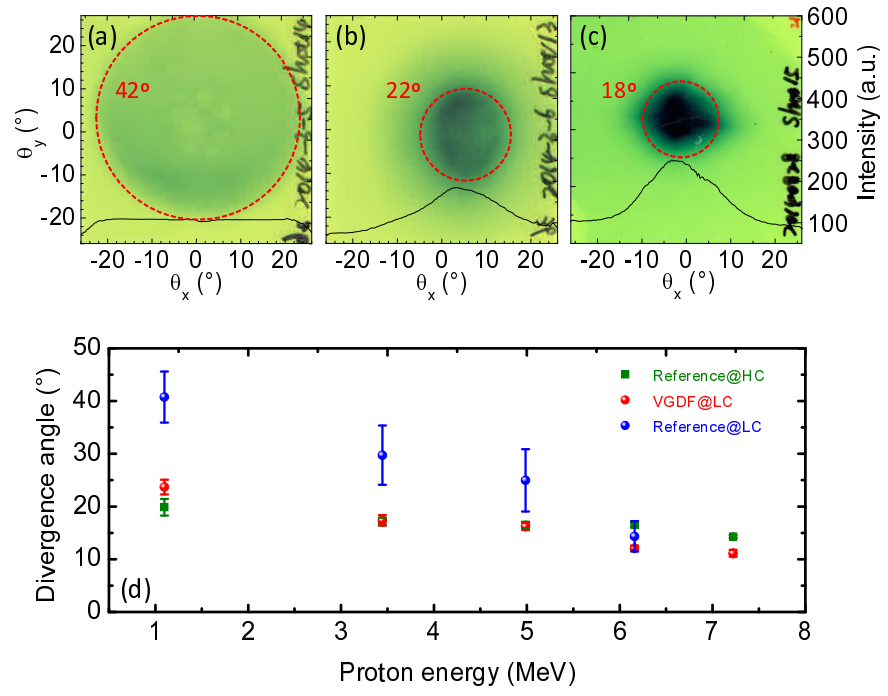

FIG. 4. (color online) Typical proton beam spatial-intensity distribution at $1.2 \mathrm{MeV}$. (a) single-layer reference target, (b) VGDF target with $L_{S F}=10 \mathrm{~nm}$ both under LC laser irradiation, and (c) single-layer reference target in $\mathrm{HC}$ case. Each red dashed circle represents the dimension of proton beam and the black line shows the line-out intensity profile along $\theta_{y}=0^{\circ}$. (d) The beam divergence angles as a function of proton energies. The errorbars correspond to shot-to-shot fluctuations and uncertainties in beam radius fitting. The SF thickness is $100 \mathrm{~nm}$ in this comparison.

reference target, corresponding to a beam divergence reduction from $42^{\circ}$ to $22^{\circ}$. The proton flux density and integrated dose from the VGDF target are also higher than the counterparts from the reference target. The darker in color and the higher value in line-out profile illustrate the larger proton number. Moreover, a denser region within a uniform area is observed from the VGDF target. It is worthy to note that the beam divergence and density flux from the VGDF target are close to those from the reference target under $\mathrm{HC}$ laser irradiation (shown in Fig. 4(c)). A smaller and darker proton beam is also observed in this case. The energy-dependent proton beam divergence for the three cases are compared in Fig. 4(d). The divergences from the VGDF target are reduced at all sampled proton energies in comparison to those from the reference target, but are coincident with those under $\mathrm{HC}$ condition. This indicates that the corresponding reduction in proton beam divergence is due to the enhancement of laser contrast.

The effect of SF thickness on laser temporal contrast characterized in proton beam divergence is investigated, as is shown in Fig. 5(a). We find that the divergence angles remain approximately constant over the range $L_{S F}=10-100 \mathrm{~nm}$ for our laser conditions. They are almost equal to that from the reference target under $\mathrm{HC}$ laser irradiation. This implies that the plasma shutter is robust in achieving high contrast laser pulse.

Although the beam divergence is not sensitive to the SF thickness, the proton acceleration has been enhanced.
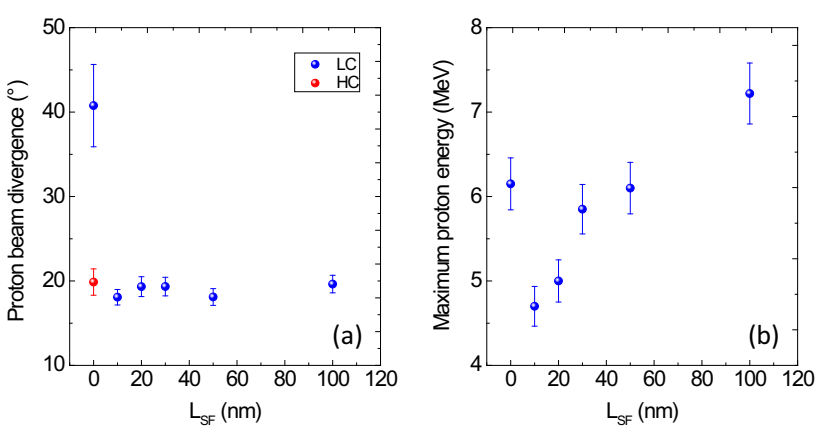

FIG. 5. (color online) (a) Proton beam divergence as a function of SF thickness under LC laser irradiation. $L_{S F}=0 \mathrm{~nm}$ represents the case without $\mathrm{SF}$, i.e., corresponding to the single-layer reference target. The red dot represents the case in $\mathrm{HC}$ case. (b) The maximum proton energy at different SF thickness in LC case.

The maximum proton energy as a function of SF thickness is presented in Fig. 5(b). After the initial drop, it monotonically increases. Higher maximum proton energy is expected when further increasing SF thickness. This can be attributed to the different density distributions of preplasma which affect the laser energy absorption and coupling to hot electrons. ${ }^{28}$ The effects may be enhanced by the density plateau formed for thicker SF which would lead to a longer interaction length. These results verify that the use of plasma shutter in VGDF target assembly allows us to actively enhance and manipulate proton beam for applications.

From the hydrodynamic simulations, the larger deformation depth of the MF rear surface will lead to the larger proton beam divergence, which is inconsistent with the constant values measured in our experiments. Hence, target deformation is not the only dominant factor determining the final proton beam divergence. To further understand the beam divergence reduction, 2D PIC simulations were performed using KLAP2D code. ${ }^{29}$ The simulation box size is $150 \mu \mathrm{m} \times 50 \mu \mathrm{m}$, with $10240 \times 5120$ cells and 20 particles per cell. The transverse density profiles are set to be uniform to exclude the influence of target deformation. The longitudinal plasma density profiles are adopted from the MULTI2D simulations shown in Fig. 2. A $p$-polarized laser pulse with $a_{0}=6.83\left(I=1 \times 10^{20}\right.$ $\mathrm{W} / \mathrm{cm}^{2}$ ) and $0.8 \mu \mathrm{m}$ wavelength is focussed at the initial front surface of MF.

Figure 6 shows the longitudinal electric field and proton density distributions for VGDF target $\left(L_{S F}=\right.$ $100 \mathrm{~nm},(\mathrm{a})$ and (c)) and reference target ((b) and (d)) at $t=200 T$, respectively. For the VGDF target, the longitudinal field is stronger and its distribution is flatter than those of reference target. The proton beam accelerated by this sheath field has a similar spatial shape, which is more uniform transversely, corresponding to a beam divergence of $\sim 10^{\circ} .{ }^{30}$ Combined with the hydrodynamic simulations and experimental results, it suggests 

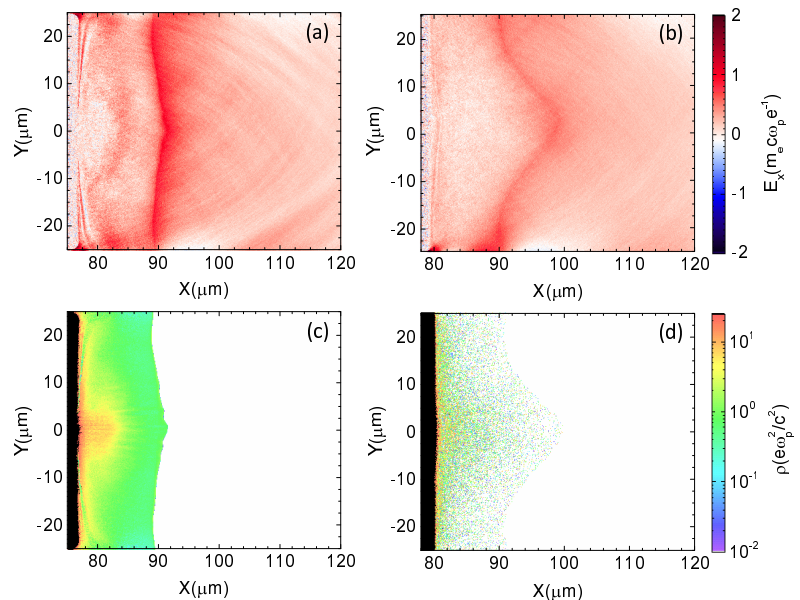

FIG. 6. (color online) Longitudinal electric field distributions at $t=200 T$ for (a) VGDF target and (b) reference target. (c) and (d) represent the corresponding proton density distributions. Laser is incident from the left-hand side. $T=$ $1 / \omega_{p} \simeq 3.33 \mathrm{fs}$.

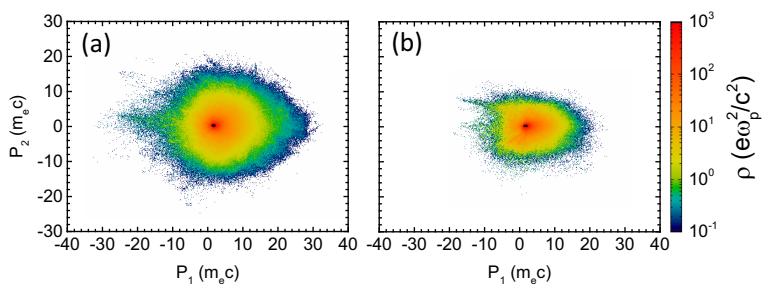

FIG. 7. (color online) The phase-space distributions of electron density for (a) VGDF target and (b) reference target at $t=50 T$ when the laser is reflected at the relativistic critical density surface.

that the decrease in proton beam divergence is a joint effect of flatter sheath field and less target deformation. A small jet-like region with higher flux density within a uniform background is observed from the VGDF target, which agrees with the experimental result shown in Fig. 4(b).

The variations in sheath field distribution may result from the diverse preplasma profiles which affect the laser absorption, fast electrons generation and transport in solid-density target etc. ${ }^{10,31,32}$ A phenomenal interpretation is that for the thick VGDF target, an underdense preplasma plateau with tens of micron is excited at the target front surface. When laser propagates into this long underdense plasma, a wakefield is generated, which traps the electron bunches and accelerates them to high energy. With the wakefield propagating into the the plasma up-ramp density region, it will suffer wave breaking and the electrons are expelled along transverse direction. As shown in Fig. 7, at $t=50 T$, electrons heated in this region obtain a higher transverse momentum from the laser ponderomotive force than that from reference target while the laser is reflected at the relativistic critical density surface. This makes the electrons inject into the overdense plasma with a larger angle, thereby producing a uniform and flat sheath field at the target rear surface which accelerates protons within a small solid angle.

\section{v. CONCLUSION}

The utility of the ultrathin foil as a plasma optical shutter has been investigated, which improves the ASE temporal contrast in a similar way as plasma mirror. The proton beam divergence as a characteristic signature of laser contrast was studied by applying the plasma shutter into a double-foil design. The reduced divergence was measured, which is attributed to the laser contrast improvement. 2D hydrodynamic and 2D PIC simulations indicate that the reduction in proton beam divergence is the combined results of the smaller target deformation and the flatter sheath field distribution.

This plasma shutter can find potential applications which requests high contrast lasers, such as HHG and ion acceleration using nano-structured or grating targets. Multiple plasma shutters can in principle be cascaded like double plasma mirrors to further improve the laser temporal contrast.

\section{ACKNOWLEDGMENTS}

We acknowledge fruitful discussions with Professor Liming Chen and Professor Paul McKenna. This work was supported by National Basic Research Program of China (grant nos. 2013CBA01500), National Natural Science Foundation of China (grant nos. 11121504, 11535001 and 11220101002), and National Key Scientific Instrument Development Project (grant nos.2012YQ030142).

${ }^{1}$ G. A. Mourou, T. Tajima and S. V. Bulanov, Rev. Mod. Phys. 782 (2006)

${ }^{2}$ T. Zh. Esirkepov, M. Borghesi, S. V. Bulanov, G. A. Mourou and

T. Tajima, Phys. Rev. Lett. 92175003 (2004).

${ }^{3}$ A. Henig, S. Steinke, M. Schnürer, T. Sokollik, R. Hörlein, D. Kiefer, D. Jung, J. Schreiber, B. M. Hegelich, X. Q. Yan, J. Meyer-ter-Vehn, T. Tajima, P. V. Nickles, W. Sandner and D. Habs, Phys. Rev. Lett. 103245003 (2009).

${ }^{4}$ L. Yin, B. J. Albright, B. M. Hegelich and J. C. Fernández, Laser Part. Beams 24291 (2006).

${ }^{5}$ M. Roth, D. Jung, K. Falk, N. Guler, O. Deppert, M. Devlin, A. Favalli, J. Fernandez, D. Gautier, M. Geissel, R. Haight, C. E. Hamilton, B. M. Hegelich, R. P. Johnson, F. Merrill, G. Schaumann, K. Schoenberg, M. Schollmeier, T. Shimada, T. Taddeucci, J. L. Tybo, F. Wagner, S. A. Wender, C. H. Wilde and G. A. Wurden, Phys. Rev. Lett. 110044802 (2013).

${ }^{6}$ C. Thaury, F. Quéré, J.-P. Geindre, A. Levy, T. Ceccotti, P. Monot, M. Bougeard, F. Réau, P. d'Oliveira, P. Audebert, R. Marjoribanks and Ph. Martin, Nat. Phys. 3 424-429 (2007). 
${ }^{7}$ M. Behmke, D. an der Brügge, C. Rödel, M. Cerchez, D. Hemmers, M. Heyer, O. Jäckel, M. Kübel, G. G. Paulus, G. Pretzler, A. Pukhov, M. Toncian, T. Toncian and O. Willi, Phys. Rev. Lett. 106185002 (2011).

${ }^{8}$ S. P. Hatchett, C. G. Brown, T. E. Cowan, E. A. Henry, J. S. Johnson, M. H. Key, J. A. Koch, A. B. Langdon, B. F. Lasinski, R. W. Lee, A. J. Mackinnon, D. M. Pennington, M. D. Perry, T. W. Phillips, M. Roth, T. C. Sangster, M. S. Singh, R. A. Snavely, M. A. Stoyer, S. C. Wilks and K. Yasuike, Phys. Plasmas 72076 (2000).

${ }^{9}$ S. C. Wilks, A. B. Langdon, T. E. Cowan, M. Roth, M. Singh, S. Hatchett, M. H. Key, D. Pennington, A. MacKinnon and R. A. Snavely, Phys. Plasmas 8542 (2001).

${ }^{10}$ J. S. Green, N. P. Dover, M. Borghesi, C. M. Brenner, F. H. Cameron, D. C. Carroll, P. S. Foster, P. Gallegos, G. Gregori, P. McKenna, C. D. Murphy, Z. Najmudin, C. A. J. Palmer, R. Prasad, L. Romagnani, K. E. Quinn, J. Schreiber, M. J. V. Streeter, S. Ter-Avetisyan, O. Tresca, M. Zepf and D. Neely, Plasma Phys. Control. Fusion 56084001 (2014).

${ }^{11}$ Y. Fang, X. L. Ge, S. Yang, W. Q. Wei, T. P. Yu, F. Liu, M. Chen, J. Q. Liu, X. H. Yuan, Z. M. Sheng and J. Zhang, Plasma Phys. Control. Fusion 58075010 (2016).

${ }^{12}$ W. P. Wang, H. Zhang, B. Wu, C. Y. Jiao, Y. C. Wu, B. Zhu, K. G. Dong, W. Hong, Y. Q. Gu, B. F. Shen, Y. Xu, Y. X. Leng, R. X. Li and Z. Z. Xu, Appl. Phys. Lett. 101214103 (2012).

${ }^{13}$ Y. Fang, T. P. Yu, X. L. Ge, S. Yang, W. Q. Wei, T. Yuan, F. Liu, M. Chen, J. Q. Liu, Y. T. Li, X. H. Yuan, Z. M. Sheng and J. Zhang, Plasma Phys. Control. Fusion 58045025 (2016).

${ }^{14}$ E. Brambrink, J. Schreiber, T. Schlegel, P. Audebert, J. Cobble, J. Fuchs, M. Hegelich and M. Roth, Phys. Rev. Lett. 96154801 (2006).

${ }^{15}$ D. C. Carroll, P. McKenna, O. Lundh, F. Lindau, C-G Wahlström, S. Bandyopadhyay, D. Pepler, D. Neely, S. Kar, P. T. Simpson, K. Markey, M. Zepf, C. Bellei, R. G. Evans, R. Redaelli, D. Batani, M. H. Xu and Y. T. Li, Phys. Rev. E 76 065401(R) (2007).

${ }^{16}$ F. Lindau, O. Lundh, A. Persson, P. McKenna, K. Osvay, D. Batani and C-G Wahlström, Phys. Rev. Lett. 95175002 (2005).

${ }^{17}$ O. Lundh, F. Lindau, A. Persson, C-G Wahlström, P. McKenna and D. Batani, Phys. Rev. E 76026404 (2007).

${ }^{18}$ G. Doumy, F. Quéré, O. Gobert, M. Perdrix, Ph. Martin, P. Audebert, J. C. Gauthier, J.-P. Geindre and T. Wittmann, Phys. Rev. E 69026402 (2004).

${ }^{19}$ A. Jullien, O. Albert, F. Burgy, G. Hamoniaux, J.-P. Rousseau,
J.-P. Chambaret, F. A.-Rochereau, G. Chériaux, J. Etchepare, N. Minkovski and S. M. Saltiel, Opt. Lett. 30 920-922 (2005).

${ }^{20}$ A. Dubietis, G. Jonusauskas and A. Piskarskas, Opt. Commun. 88437 (1992).

${ }^{21}$ M. P. Kalashnikov, E. Risse, H. Schönnagel, A. Husakou, J. Herrmann and W. Sandner, Opt. Express 12 5088-5097 (2004).

${ }^{22}$ V. A. Vshivkov, N. M. Naumova, F. Pegoraro and S. V. Bulanov, Phys. Plasmas 52727 (1998).

${ }^{23}$ S. A. Reed, T. Matsuoka, S. Bulanov, M. Tampo, V. Chvykov, G. Kalintchenko, P. Rousseau, V. Yanovsky, R. Kodama, D. W. Litzenberg, K. Krushelnick and A. Maksimchuk, Appl. Phys. Lett. 94201117 (2009).

${ }^{24}$ S. Palaniyappan, B. M. Hegelich, H. C. Wu, D. Jung, D. C. Gautier, L. Yin, B. J. Albright, R. P. Johnson, T. Shimada, S. Letzring, D. T. Offermann, J. Ren, C. K. Huang, R. Hörlein, B. Dromey, J. C. Fernandez and R. C. Shah, Nat. Phys. 8763 C769 (2012).

${ }^{25}$ B. G. Izquierdo, R. J. Gray, M. King, R. J. Dance, R. Wilson, J. McCreadie, N. M. H. Butler, R. Capdessus, S. Hawkes, J. S. Green, M. Borghesi, D. Neely and P. McKenna, Nat. Phys. 12 505 C512 (2016).

${ }^{26}$ R. Ramis, K.Eidmann, J. Meyer-ter-Vehn and S. Hüller, Comp. Phys. Commun. 183 637-655 (2012).

${ }^{27}$ R. Ramis, J. Meyer-ter-Vehn and J. Ramíreza, Comp. Phys. Commun. 180 977-994 (2009).

${ }^{28}$ R. J. Gray, D. C. Carroll, X. H. Yuan, C. M. Brenner, M. Burza, M. Coury, K. L. Lancaster, X. X. Lin, Y. T. Li, D. Neely, M. N. Quinn, O. Tresca, C-G Wahlström and P. McKenna, New J. Phys. 16113075 (2014).

${ }^{29}$ M. Chen, Z. M. Sheng, Y. Y. Ma and J. Zhang, Chin. J. Comp. Phys. 25 43-50 (2008).

${ }^{30}$ A. Andreev, T. Ceccotti, A. Levy, K. Platonov and Ph. Martin, New J. Phys. 12045007 (2010).

${ }^{31}$ H. B. Cai, K. Mima, A. Sunahara, T. Johzaki, H. Nagatomo, S. P. Zhu and X. T. He, Phys. Plasmas 17023106 (2010).

${ }^{32}$ T. Zh. Esirkepov, J. K. Koga, A. Sunahara, T. Morita, M. Nishikino, K. Kageyama, H. Nagatomo, K. Nishihara, A. Sagisaka, H. Kotaki, T. Nakamura, Y. Fukuda, H. Okada, A. S. Pirozhkov, A. Yogo, M. Nishiuchi, H. Kiriyama, K. Kondo, M. Kando and S. V. Bulanov, Nucl. Instrum. Meth. Phys. Res. A 745 150C163 (2014).

${ }^{33}$ B. S. Paradkar, M. S. Wei, T. Yabuuchi, R. B. Stephens, M. G. Haines, S. I. Krasheninnikov and F. N. Beg, Phys. Rev. E 83 046401 (2011). 